\title{
POSTULATE-SETS FOR BOOLEAN RINGS
}

\author{
BY \\ B. A. BERNSTEIN(1)
}

1. Introduction. Boolean rings (or generalized Boolean algebras), defined by Stone, are rings in which every element is idempotent. Sets of postulates for these rings have been given by Stone $\left({ }^{2}\right)$ and by Stabler $\left({ }^{3}\right)$. I give in this paper a number of additional postulate-sets for such rings. The sets are all expressed in terms of ring addition and multiplication. Stone's postulates for Boolean rings in terms of ring operations are given as set $S$ below; Stabler's postulates of this type are sets $S^{\prime}$ and $S^{\prime \prime}$ below. The additional sets of the present paper are sets I-IX below.

Stone has not undertaken the task of eliminating possible redundancies from his set $S$, and Stabler raised the question of independence of his set $S^{\prime}$ when a unit-element postulate is added to $S^{\prime}$. It will be shown that, except for an overlooked redundancy, set $S$ is independent and that $S^{\prime}$ remains independent after the addition of a unit-element postulate. The latter fact will be shown to hold also for set $S$, without the redundancy, and for sets $S^{\prime \prime}$, I-IX.

2. List of postulates. The postulates used in the various sets will be found in the list of conditions below on a class $K$ and two binary operations,$+ X$. (In the postulates that are not existence postulates supply the restriction: if the elements indicated are in $K$.) The list of postulates follows.

N. $K$ is non-empty.

$\mathrm{N}^{\prime}$. If $a$ is an element of $K$, there is an element $b$ in $K$ such that $b \neq a$.

$\mathrm{A}_{1} . a+b$ is in $K$ whenever $a, b$ are in $K$.

A. $a+(b+c)=(a+b)+c$.

$\mathrm{A}_{2}^{\prime} \cdot a+(b+c)=b+(c+a)$.

$\mathrm{A}_{2}^{\prime \prime} \cdot a+(b+c)=c+(b+a)$.

$A_{3}$. For any two elements $a, b$ in $K$ there is an element $x$ in $K$ such that $x+a=b$.

$\mathbf{A}_{3}^{\prime}$. For any two elements $a, b$ in $K$ there is an element $x$ in $K$ such that $x+a=b$ and $a+x=b$.

$\mathrm{A}_{3}^{\prime \prime}$. For any two elements $a, b$ in $K$ there is an element $y$ in $K$ such that $a+y=b$.

Presented to the Society, April 24, 1943; received by the editors August 12, 1943.

(1) I am indebted to the referee for valuable changes made in the manuscript.

(2) M. H. Stone, The theory of representations for Boolean algebras, Trans. Amer. Math. Soc. vol. 40 (1936) pp. 37-111, especially pp. 39, 40; Postulates for Boolean algebras and generalized Boolean algebras, Amer. J. Math. vol. 57 (1935) pp. 703-732.

(') E. R. Stabler, Sets of postulates for Boolean rings, Amer. Math. Monthly vol. 48 (1941) pp. 21-28. 
A4. $a+b=b+a$.

A. If $a+b_{1}=a+b_{2}$ then $b_{1}=b_{2}$.

$\mathrm{A}_{5}^{\prime}$. If $a_{1}+b=a_{2}+b$ then $a_{1}=a_{2}$.

A6. $(a+a)+b=b$.

$\mathrm{A}_{6}^{\prime} \cdot b+(a+b)=a$.

$\mathrm{A}_{6}^{\prime \prime} \cdot a+(a+b)=b$.

$\mathrm{A}_{6}^{\prime \prime \prime} .(a+b)+b=a$.

A . If $a+b=c$ then $b+c=a$.

$\mathrm{M}_{1} . a b$ is in $K$ whenever $a, b$ are in $K$.

$\mathrm{M}_{2} \cdot a(b c)=(a b) c$.

$\mathbf{M}_{2}^{\prime} \cdot a(b c)=b(c a)$.

$\mathrm{M}_{2}^{\prime \prime} \cdot a(a b)=(a a) b$.

$\mathrm{M}_{2}^{\prime \prime \prime} . a(b c)=c(b a)$.

$\mathrm{M}_{3} \cdot a(b+c)=a b+a c$.

$\mathbf{M}_{3}^{\prime} \cdot(a+b) c=a c+b c$.

M. $a a=a$.

M. $P_{1}+\left\{P_{2}+\left[P_{3}+\left(P_{4}+P_{5}\right)\right]\right\}=g+g$, where $P_{1}=[a+(b+c)]+[b$ $+(c+a)], P_{2}=c(d e)+d(e c), P_{3}=a(d+f)+(a d+a f), P_{4}=b b+b, P_{5}=c(c+c)$.

$\mathrm{M}_{6}$. If $K$ is not empty, there is an element 1 in $K$ such that either $1 a=a$ for every element $a$ in $K$ or $a 1=a$ for every element $a$ in $K$.

$\mathrm{M}_{6}^{\prime}$. If $K$ is not empty, there is an element 1 in $K$ such that $1 a=a$ for every element $a$ in $K$.

3. Sets $\mathbf{S}, \mathbf{S}^{\prime}, \mathbf{S}^{\prime \prime}$. Following are sets $S, S^{\prime}, S^{\prime \prime}$.

Set $S: N, A_{1}, A_{2}, A_{3}, A_{4}, M_{1}, M_{2}, M_{3}, M_{3}^{\prime}, M_{4}$.

Set $S^{\prime}: N, A_{1}, A_{2}, A_{5}, A_{6}^{\prime}, M_{1}, M_{2}, M_{3}, M_{3}^{\prime}, M_{4}$.

Set $S^{\prime \prime}: N, A_{1}, A_{2}^{\prime}, A_{6}, M_{1}, M_{2}^{\prime}, M_{3}, M_{4}$.

It will be observed that set $S$ embeds a set of postulates for abelian groups as well as a set for general rings. Set $S^{\prime}$ is set $S$ in which $A_{3}, A_{4}$ are replaced by the symmetric pair of cancellation laws $A_{5}, A_{5}^{\prime}$. It thus embeds postulates for a semi-group and is the weakest of the three sets. Stabler found $S^{\prime \prime}$ as a subset in a set of postulates given by me for Boolean algebra $\left({ }^{4}\right)$. It embeds postulates for Boolean groups $\left({ }^{5}\right)$, and is the briefest and strongest of the three sets. It has cyclic associative laws, instead of the ordinary associative laws, an unconditioned cancellation postulate, and only one distributive law. All the postulates of $S^{\prime \prime}$, except the postulates of non-emptiness and closure, are equivalence postulates.

4. Sets I-IX. The sets I-IX follow.

Set I: $N, A_{2}, A_{3}^{\prime}, M_{1}, M_{2}, M_{3}, M_{3}^{\prime}, M_{4}$.

Set II: $N, A_{1}, A_{2}, A_{5}, A_{5}^{\prime}, M_{1}, M_{2}^{\prime \prime}, M_{3}, M_{3}^{\prime}, M_{4}$.

(4) B. A. Bernstein, Postulates for Boolean algebra involving the operation of complete disjunction, Ann. of Math. (2) vol. 37 (1936) pp. 317-325.

(5) See my Sets of postulates for Boolean groups, Ann. of Math. (2) vol. 40 (1939) pp. 420 422. A Boolean group is an abelian group in which every element is its own inverse. 
Set III : N, $A_{1}, A_{2}^{\prime}, A_{6}^{\prime \prime}, M_{1}, M_{2}^{\prime}, M_{3}, M_{4}$.

Set IV: $N, A_{1}, A_{2}, A_{6}^{\prime \prime}, A_{6}^{\prime \prime \prime}, M_{1}, M_{2}, M_{3}, M_{3}^{\prime}, M_{4}$.

Set V: N, $A_{1}, A_{2}, A_{6}^{\prime}, M_{1}, M_{2}, M_{3}, M_{3}^{\prime}, M_{4}$.

Set VI: $N, A_{1}, A_{2}, A_{7}, M_{1}, M_{2}, M_{3}, M_{3}^{\prime}, M_{4}$.

Set VII: N, $A_{2}, A_{3}, A_{3}^{\prime \prime}, M_{1}, M_{2}, M_{3}, M_{3}^{\prime}, M_{4}$.

Set VIII: N, $A_{1}, A_{2}^{\prime \prime}, A_{6}^{\prime \prime}, M_{1}, M_{2}^{\prime \prime \prime}, M_{3}, M_{3}^{\prime}, M_{4}$.

Set IX: N, $A_{1}, A_{7}, M_{1}, M_{5}$.

In sets I-VIII the aim is simplicity and symmetry and "naturalness." Set $I$ is a slight variation of set $S$. Like set $S$, it contains a set of postulates for abelian groups (due to Garver) and a set for general rings, but it is a little more symmetric than set $\mathrm{S}$.

Set II is set $\mathbf{S}^{\prime}$ in which the multiplicative associative law is replaced by one involving only two arguments. It thus answers in the affirmative a question that naturally suggests itself concerning set $S^{\prime}$ : Can this very weak set be weakened in any way?

Sets III-VI are of type $\mathrm{S}^{\prime \prime}$; each embeds a set of postulates for Boolean groups. Set III is set $S^{\prime \prime}$ in which the (unconditioned) cancellation postulate $A_{6}$ is replaced by $A_{6}^{\prime \prime}$. Set IV has a symmetric pair of cancellation postulates, $\mathrm{A}_{6}^{\prime \prime}$ and $\mathrm{A}_{6}^{\prime \prime \prime}$, the ordinary associative laws, and both right and left distributive laws. Set $V$ is $I V$ with the pair $A_{6}^{\prime \prime}, A_{6}^{\prime \prime \prime}$ replaced by the single $A_{6}^{\prime}$. Set VI is IV with $\mathbf{A}_{6}^{\prime \prime}, \mathbf{A}_{6}^{\prime \prime \prime}$ replaced by the cyclic transposition law $\mathbf{A}_{7}$.

Set VII embeds Garver's postulates for groups $\left({ }^{6}\right)$. Set VIII embeds a set of postulates for subtractive abelian groups $\left({ }^{7}\right)$. In sets III-V, VIII all the postulates, except $\mathrm{N}, \mathrm{A}_{1}, \mathrm{M}_{1}$, are equivalence postulates.

Set IX is very different from the other sets. It is a very condensed set. Postulates $M_{5}$ of this set is a condensation of the equivalence postulates $\mathrm{A}_{2}^{\prime}, \mathrm{M}_{2}^{\prime}, \mathrm{M}_{3}, \mathrm{M}_{4}$, and the identity $a(a+a)=0$. It will be observed that this condensation is effected almost mechanically, and that the general procedure employed in the condensation is applicable to many systems other than Boolean rings.

5. Consistency and necessity of sets I-IX. All the postulates in sets I-IX are properties of Boolean rings. Sets I-IX are thus consistent, and necessary for Boolean rings.

6. Sufficiency of sets I, III-VII. The sufficiency of each of the Sets I, III-VII for Boolean rings is clear.

7. Sufficiency of set II. Theorems 7.1-7.5 following prove the sufficiency of set II for Boolean rings.

\section{1. $K$ is a Boolean group with respect to + .}

7.2. $a b=b a$.

(6) Raymond Garver, Bull. Amer. Math. Soc. vol. 42 (1936) pp. 125-129.

(7) See my Postulates for abelian groups and fields in terms of non-associative operations, Trans. Amer. Math. Soc. vol. 43 (1938) pp. 1-6, especially p. 5. 
Theorems 7.1 and 7.2 are derivable from set II, since these theorems are derivable from set $\mathrm{S}^{\prime}$ without $\mathbf{M}_{\mathbf{2}}$ (see Stabler, loc. cit.). The proof of 7.2 follows. $a+b=(a+b)(a+b)=(a+b) a+(a+b) b=(a a+b a)+(a b+b b)=(a+b a)$ $+(a b+b)$, by $\mathbf{M}_{4}, \mathbf{M}_{3}, \mathbf{M}_{3}^{\prime}, \mathbf{M}_{4}$. Hence $a b+b a=0$, by 7.1. Hence $a b=b a$, by 7.1 .

7.3. $a b=b a=b(a a)=(a a) b=a(a b)=a(b a)$.

This is proved by using 7.2, $\mathrm{M}_{4}, 7.2, \mathrm{M}_{2}^{\prime \prime}, 7.2$.

7.4. $(a+b)(a b)=0$.

This is proved by using $M_{3}^{\prime}, 7.3,7.1$.

7.5. $a(b c)=(a b) c$.

For,

$$
\begin{aligned}
0 & =[(a+b)+(b+c)][(a+b)(b+c)]=(a+c)[(a+b)(b+c)] \\
& =(a+c)[a(b+c)+b(b+c)]=(a+c)[(a b+a c)+(b b+b c)] \\
& =a[(a b+a c)+(b b+b c)]+c[(a b+a c)+(b b+b c)] \\
& =[a(a b+a c)+a(b b+b c)]+[c(a b+a c)+c(b b+b c)] \\
& =\{[a(a b)+a(a c)]+[a(b b)+a(b c)]\}+\{[c(a b)+c(a c)]+[c(b b)+c(b c)]\} \\
& =\{(a b+a c)+[a b+a(b c)]\}+\{c(a b)+a c]+(b c+b c)\} \\
& =a(b c)+c(a b)=a(b c)+(a b) c,
\end{aligned}
$$

by 7.4, 7.1, $\mathrm{M}_{3}^{\prime}, \mathrm{M}_{3}, \mathrm{M}_{3}^{\prime}, \mathrm{M}_{3}, \mathrm{M}_{3}, 7.3,7.1,7.2$. Therefore $a(b c)=(a b) c$, by 7.1.

8. Sufficiency of set VIII. The sufficiency of set VIII for Boolean rings will be proved by showing that set VIII yields set $S$.

Let $\oplus$ be the direct group operation. In view of postulates $N, A_{1}, A_{2}^{\prime \prime}, A_{6}^{\prime \prime}$ and the author's paper referred to in footnote $7, K$ is an abelian group in which + is the operation of subtraction, with the understanding that $a \oplus b$ is defined by $a \oplus b=a+b$ where $b=(a+a)+b$. In order to prove that VIII yields $\mathrm{S}$, it will suffice to prove that $K$ is also an additive abelian group with respect to + and that the operation $X$ is associative. The proofs are given by Theorems 8.1-8.6 following. (The proofs of 8.1, 8.2, 8.4, 8.6 are indicated by the bracketed references.)

8.1. $K$ is a subtractive abelian group with respect to + , with $a+a$ as 0 . $\left[\mathrm{N}, \mathrm{A}_{1}, \mathrm{~A}_{2}^{\prime \prime}, \mathrm{A}_{6}^{\prime \prime}\right]$

8.2. $(a+a) b=b(a+a)=0$. $\left[\mathrm{M}_{3}^{\prime}, \mathrm{M}_{3}, 8.1\right]$

8.3. $(a+a)+b=b$.

For, $(a+a)+b=[(a+a)+b][(a+a)+b]=(a+a)[(a+a)+b]+b[(a+a)+b]$ 
$=(a+a)[(a+a)+b]+[b(a+a)+b b]=0+(0+b b)=b b=b$, by $\mathbf{M}_{4}, \mathbf{M}_{3}^{\prime}, \mathbf{M}_{3}, 8.2$, $\mathrm{A}_{6}^{\prime \prime}, \mathrm{M}_{4}$. 8.3]

8.4. $K$ is an additive abelian group with respect to + , with $a+a=0 .[8.1$,

8.5. $a b=b a$.

See the proof of 7.2 above.

8.6. $a(b c)=(a b) c .\left[\mathrm{M}_{2}^{\prime}\right.$, , 8.5]

Theorems 8.4, 8.6, and postulates $N, M_{1}, M_{3}, M_{3}^{\prime}, M_{4}$ give set $S$.

9. Sufficiency of set IX. The following theorems from set IX, together with postulates $N, A_{1}, M_{1}$, give set $S^{\prime \prime}$.

9.1. $a+a=b+b .\left[\mathrm{M}_{5}\right]$

9.2. Definition. $0=a+a$.

9.3. $0+a=a+0=a .\left[9.2, \mathrm{~A}_{7}, \mathrm{~A}_{7}\right]$

9.4. $P_{1}+\left\{P_{2}+\left[P_{3}+\left(P_{4}+P_{5}\right)\right]\right\}=0$. $\left[\mathrm{M}_{5}, 9.2\right]$

9.5. $P_{2}+\left[P_{3}+\left(P_{4}+P_{5}\right)\right]=0 .[9.4$ for $a=b=c, 9.2,9.3]$

9.6. $P_{1}=0 .[9.4,9.5,9.3]$

9.7. $P_{8}+\left(P_{4}+P_{5}\right)=0$. $[9.5$ for $c=d=e, 9.2,9.3]$

9.8. $\quad P_{2}=0 .[9.5,9.7,9.3]$

9.9. $P_{3}=0$. $[9.7$ for $b=c=0,9.3,9.2,9.3]$

9.10. $P_{4}+P_{6}=0 .[9.7,9.9,9.3]$

9.11. $a 0=0$. [9.9 for $d=f, 9.2,9.3$ ]

9.12. $P_{5}=0$. $[9.2,9.11]$

9.13. $P_{4}=0 .[9.10,9.12,9.3]$

9.14. $(a+a)+b=b .[9.2,9.3]$

9.15. If $a+b=0$ then $a=b$. $\left[\mathrm{A}_{7}, 9.3\right]$

10. Relation to Boolean algebras. A Boolean ring with unit is a Boolean algebra. Accordingly, each of the sets S-IX determines a Boolean algebra if Postulate $M_{6}$ (or $M_{6}^{\prime}$ in the case of set $S^{\prime \prime}$ ) of $\$ 2$ be added to the set. It must be noted, however, that the algebra determined by the enlarged sets is a Boolean algebra only in the sense of Stone, who would call a one-element Boolean ring a Boolean algebra. Such a Boolean algebra cannot yield the proposition of traditional logic: $a^{\prime} \neq a$, "the contradictory of a proposition $a$ 
cannot be equivalent to $a . "$ If sets of postulates for Boolean algebras in the older sense be desired, add to each of the enlarged sets Postulate $\mathrm{N}^{\prime}$ of $\S 2$.

11. Independence. Sets $S^{\prime}$ and $S^{\prime \prime}$ have been proved independent. Set $S$ is not independent. Postulate $A_{1}$ of set $S$ is redundant, in view of Garver's postulates for groups. Set $S$, without $A_{1}$, and sets I-IX are each independent. Moreover, each of these sets remains independent when enlarged by the unitelement postulate $\mathbf{M}_{6}$. Independence proofs for these enlarged sets will be given in $\$ 13$.

12. List of independence-systems. The various independence-systems employed are taken from the list of concrete systems $(K, \oplus, \odot)$ below. In these systems,$+ \vee$ denote respectively arithmetic addition and logical addition. In systems vi and ix the $\oplus$-tables define Boolean ring addition. System $\mathrm{xxi}$ is due to Stone. The list of systems follows.

i. $K$ a null class.

ii. $K=0,1,2 ; a \oplus b=2(a+b) \bmod 3 ; a \odot b=b$.

iii. $K=0,1 ; a \oplus b=a \bigvee b ; a \odot b=a b$.

iv. $K=0,1 ; a \oplus b=a ; a \odot b=a$.

v. $K=0,1 ; a \oplus b=a+b \bmod 2 ; a \odot b=a b$, except that $0 \odot 0$ is not in $K$.

vi. $K=0,1, \alpha, \alpha^{\prime}, \beta, \beta^{\prime}, \gamma, \gamma^{\prime} ; a \oplus b, a \odot b$ defined by

\begin{tabular}{c|cccccccc}
$\oplus$ & 0 & 1 & $\alpha$ & $\alpha^{\prime}$ & $\beta$ & $\beta^{\prime}$ & $\gamma$ & $\gamma^{\prime}$ \\
\hline 0 & 0 & 1 & $\alpha$ & $\alpha^{\prime}$ & $\beta$ & $\beta^{\prime}$ & $\gamma$ & $\gamma^{\prime}$ \\
1 & 1 & 0 & $\alpha^{\prime}$ & $\alpha$ & $\beta^{\prime}$ & $\beta$ & $\gamma^{\prime}$ & $\gamma$ \\
$\alpha$ & $\alpha$ & $\alpha^{\prime}$ & 0 & 1 & $\gamma$ & $\gamma^{\prime}$ & $\beta$ & $\beta^{\prime}$ \\
$\alpha^{\prime}$ & $\alpha^{\prime}$ & $\alpha$ & 1 & 0 & $\gamma^{\prime}$ & $\gamma$ & $\beta^{\prime}$ & $\beta$ \\
$\beta$ & $\beta$ & $\beta^{\prime}$ & $\gamma$ & $\gamma^{\prime}$ & 0 & 1 & $\alpha$ & $\alpha^{\prime}$ \\
$\beta^{\prime}$ & $\beta^{\prime}$ & $\beta$ & $\gamma^{\prime}$ & $\gamma$ & 1 & 0 & $\alpha^{\prime}$ & $\alpha$ \\
$\gamma$ & $\gamma$ & $\gamma^{\prime}$ & $\beta$ & $\beta^{\prime}$ & $\alpha$ & $\alpha^{\prime}$ & 0 & 1 \\
$\gamma^{\prime}$ & $\gamma^{\prime}$ & $\gamma$ & $\beta^{\prime}$ & $\beta$ & $\alpha^{\prime}$ & $\alpha$ & 1 & 0
\end{tabular}

\begin{tabular}{c|cccccccc}
$\odot$ & 0 & 1 & $\alpha$ & $\alpha^{\prime}$ & $\beta$ & $\beta^{\prime}$ & $\gamma$ & $\gamma^{\prime}$ \\
\hline 0 & 0 & 0 & 0 & 0 & 0 & 0 & 0 & 0 \\
1 & 0 & 1 & $\alpha$ & $\alpha^{\prime}$ & $\beta$ & $\beta^{\prime}$ & $\gamma$ & $\gamma^{\prime}$ \\
$\alpha$ & 0 & $\alpha$ & $\alpha$ & 0 & 1 & $\alpha^{\prime}$ & $\alpha^{\prime}$ & 1 \\
$\alpha^{\prime}$ & 0 & $\alpha^{\prime}$ & 0 & $\alpha^{\prime}$ & $\beta^{\prime}$ & $\gamma$ & $\beta^{\prime}$ & $\gamma$ \\
$\beta$ & 0 & $\beta$ & 1 & $\beta^{\prime}$ & $\beta$ & 0 & $\beta^{\prime}$ & 1 \\
$\beta^{\prime}$ & 0 & $\beta^{\prime}$ & $\alpha^{\prime}$ & $\gamma$ & 0 & $\beta^{\prime}$ & $\alpha^{\prime}$ & $\gamma$ \\
$\gamma$ & 0 & $\gamma$ & $\alpha^{\prime}$ & $\beta^{\prime}$ & $\beta^{\prime}$ & $\alpha^{\prime}$ & $\gamma$ & 0 \\
$\gamma^{\prime}$ & 0 & $\gamma^{\prime}$ & 1 & $\gamma$ & 1 & $\gamma$ & 0 & $\gamma^{\prime}$.
\end{tabular}

vii. $K=0,1, \alpha, \alpha^{\prime} ; a \oplus b$ as in ix; $a \odot 0=0, a \odot b=a(b \neq 0)$. viii. $K=0,1, \alpha, \alpha^{\prime} ; a \oplus b$ as in ix; $0 \odot b=0, a \odot b=b(a \neq 0)$.

ix. $K=0,1, \alpha, \alpha^{\prime} ; a \oplus b, a \odot b$ defined by

\begin{tabular}{c|cccc}
$\oplus$ & 0 & 1 & $\alpha$ & $\alpha^{\prime}$ \\
\hline 0 & 0 & 1 & $\alpha$ & $\alpha^{\prime}$ \\
1 & 1 & 0 & $\alpha^{\prime}$ & $\alpha$ \\
$\alpha$ & $\alpha$ & $\alpha^{\prime}$ & 0 & 1 \\
$\alpha^{\prime}$ & $\alpha^{\prime}$ & $\alpha$ & 1 & 0,
\end{tabular}

\begin{tabular}{l|llll}
$\odot$ & 0 & 1 & $\alpha$ & $\alpha^{\prime}$ \\
\hline 0 & 0 & 0 & 0 & 0 \\
1 & 0 & 1 & $\alpha$ & $\alpha^{\prime}$ \\
$\alpha$ & 0 & $\alpha$ & 1 & $\alpha^{\prime}$ \\
$\alpha^{\prime}$ & 0 & $\alpha^{\prime}$ & $\alpha^{\prime}$ & 0.
\end{tabular}

x. $K=0,1 ; a \oplus b=a+b \bmod 2$, except that $1 \oplus 1$ is not in $K ; a \odot b=a b$. 
xi. $K=0,1,2 ; a \oplus b=2(a+b) \bmod 3 ; a \odot b=a$.

xii. $K=0,1 ; a \oplus b=a ; a \odot b=a b$.

xiii. $K=0,1 ; a \oplus b=b ; a \odot b=a b$.

xiv. $K=0,1,2 ; a \oplus b=a+b \bmod 3 ; a \odot 0=0, a \odot b=a(b \neq 0)$.

xv. $K=0,1,2 ; a \oplus b=a+b \bmod 3 ; 0 \odot b=0, a \odot b=b(a \neq 0)$.

xvi. $K=0,1 ; a \oplus b=a+b \bmod 2 ; a \odot b=b$.

xvii. $K=0,1 ; a \oplus b=a+b \bmod 2 ; a \odot b=a \vee b$.

xviii. $K=0,1 ; a \oplus b=b ; a \odot b=b$.

xix. $K=0,1,2 ; a \oplus b=a+b \bmod 3 ; a \odot b=a b \bmod 3$.

xx. $K=0,1 ; a \oplus b=0 ; a \odot b=a b$.

xxi. $K$ is the set of all proper subsets of a given infinite set; $a \oplus b$ is the symmetric difference of $a, b ; a \odot b$ is the intersection of $a, b$.

13. Independence proofs. Let $S_{1}$ denote set $S$ without Postulate $A_{1}$. Proofs of independence for $S_{1}, S^{\prime}, \cdots, I X$, with $M_{6}$ added to each set, can now be given. Below are listed these enlarged sets, each coupled with a set of systems (from \$12) establishing the independence of its postulates. In each pair of postulate-set and independence-system set the order of the postulates is that of the corresponding independence-systems. The enlarged sets are labelled $\overline{\mathrm{S}}_{1}, \overline{\mathrm{S}}^{\prime}, \cdots, \overline{\mathrm{IX}}$, the corresponding sets of independence-systems are $K \overline{\mathrm{S}}_{1}, K \overline{\mathrm{S}}^{\prime}, \cdots, K \overline{\mathrm{IX}}$. Remarks on the independence proofs follow the listed pairs of postulate-sets and system-sets. Systems $K \overline{\mathrm{S}}^{\prime \prime}$ will also serve as independence-systems for the set obtained from $\bar{S}^{\prime \prime}$ by replacing $M_{6}$ by $M_{6}^{\prime}$.

$\bar{S}_{1}: N, A_{2}, A_{3}, A_{4}, M_{1}, M_{2}, M_{3}, M_{3}^{\prime}, M_{4}, M_{6}$.

$K \overline{\mathrm{S}}_{1}$ : i, ii, iii, iv, v, vi, vii, viii, ix, xxi.

$\bar{S}^{\prime}: N, A_{1}, A_{2}, A_{5}, A_{6}^{\prime}, M_{1}, M_{2}, M_{3}, M_{3}^{\prime}, M_{4}, M_{6}$.

$\left.K \overline{\mathrm{S}}^{\prime}: \mathrm{i}, \mathrm{x}, \mathrm{xi}{ }^{8}\right)$, xii, xiii, v, vi, xiv, $\mathrm{xv}, \mathrm{ix}, \mathrm{xxi}$.

$\overline{\mathrm{S}}^{\prime \prime}: \mathrm{N}, \mathrm{A}_{1}, \mathrm{~A}_{2}^{\prime}, \mathrm{A}_{6}, \mathrm{M}_{1}, \mathrm{M}_{2}^{\prime}, \mathrm{M}_{3}, \mathrm{M}_{4}, \mathrm{M}_{6}$.

$K \overline{\mathrm{S}}^{\prime \prime}: \mathrm{i}, \mathrm{x}, \mathrm{xiii}, \mathrm{iii}, \mathrm{v}, \mathrm{xvi}, \mathrm{xvii}, \mathrm{ix}, \mathrm{xxi}$.

$\overline{\mathrm{I}}: \mathrm{N}, \mathrm{A}_{2}, \mathrm{~A}_{3}^{\prime}, \mathrm{M}_{1}, \mathrm{M}_{2}, \mathrm{M}_{3}, \mathrm{M}_{3}^{\prime}, \mathrm{M}_{4}, \mathrm{M}_{6}$.

$K \overline{\mathrm{I}}: \mathrm{i}$, ii, iii, v, vi, vii, viii, ix, xxi.

$\overline{I I}: \mathrm{N}, \mathrm{A}_{1}, \mathrm{~A}_{2}, \mathrm{~A}_{5}, \mathrm{~A}_{5}^{\prime}, \mathrm{M}_{1}, \mathrm{M}_{2}^{\prime \prime}, \mathrm{M}_{3}, \mathrm{M}_{3}^{\prime}, \mathrm{M}_{4}, \mathrm{M}_{6}$.

$K \overline{\mathrm{II}}$ : $\mathrm{i}, \mathrm{x}, \mathrm{xi}, \mathrm{xii}, \mathrm{xiii}, \mathrm{v}, \mathrm{vi}, \mathrm{xiv}, \mathrm{xv}, \mathrm{ix}, \mathrm{xxi}$.

$\overline{\mathrm{III}}: \mathrm{N}, \mathrm{A}_{1}, \mathrm{~A}_{2}^{\prime}, \mathrm{A}_{6}^{\prime \prime}, \mathrm{M}_{1}, \mathrm{M}_{2}^{\prime}, \mathrm{M}_{3}, \mathrm{M}_{4}, \mathrm{M}_{6}$.

$K \overline{I I I}: \mathrm{i}, \mathrm{x}, \mathrm{xiii}, \mathrm{iii}, \mathrm{v}, \mathrm{xvi}, \mathrm{xvii}, \mathrm{ix}, \mathrm{xxi}$.

$\overline{I V}: \mathrm{N}, \mathrm{A}_{1}, \mathrm{~A}_{2}, \mathrm{~A}_{6}^{\prime \prime}, \mathrm{A}_{6}^{\prime \prime \prime}, \mathrm{M}_{1}, \mathrm{M}_{2}, \mathrm{M}_{3}, \mathrm{M}_{3}^{\prime}, \mathrm{M}_{4}, \mathrm{M}_{6}$.

$K \overline{\mathrm{IV}}: \mathrm{i}, \mathrm{x}, \mathrm{ii}, \mathrm{iv}, \mathrm{xviii}, \mathrm{v}$, vi, vii, viii, ix, xxi.

$\bar{V}: \mathrm{N}, \mathrm{A}_{1}, \mathrm{~A}_{2}, \mathrm{~A}_{6}^{\prime}, \mathrm{M}_{1}, \mathrm{M}_{2}, \mathrm{M}_{3}, \mathrm{M}_{3}^{\prime}, \mathrm{M}_{4}, \mathrm{M}_{6}$.

$K \overline{\mathrm{V}}: \mathrm{i}, \mathrm{x}, \mathrm{ii}, \mathrm{iv}, \mathrm{v}, \mathrm{vi}$, vii, viii, ix, xxi.

$\overline{\mathrm{VI}}: \mathrm{N}, \mathrm{A}_{1}, \mathrm{~A}_{2}, \mathrm{~A}_{7}, \mathrm{M}_{1}, \mathrm{M}_{2}, \mathrm{M}_{3}, \mathrm{M}_{3}^{\prime}, \mathrm{M}_{4}, \mathrm{M}_{6}$.

(8) System ii, used as independence-system for $A_{2}$ in other sets, may replace system xi. This fact was pointed out to me by the referee. System xi was retained to avoid the general renumbering of the independence systems and so avoid the risk of introducing serious typographical errors in the paper. 
$K \overline{\mathrm{VI}}: \mathrm{i}, \mathrm{x}, \mathrm{ii}, \mathrm{iii}, \mathrm{v}, \mathrm{vi}, \mathrm{vii}, \mathrm{viii}, \mathrm{ix}, \mathrm{xxi}$.

$\overline{V I I}: \mathrm{N}, \mathrm{A}_{2}, \mathrm{~A}_{3}, \mathrm{~A}_{8}^{\prime \prime}, \mathrm{M}_{1}, \mathrm{M}_{2}, \mathrm{M}_{3}, \mathrm{M}_{8}^{\prime}, \mathrm{M}_{4}, \mathrm{M}_{6}$.

$K \overline{\mathrm{VII}}: \mathrm{i}$, ii, xviii, iv, v, vi, vii, viii, ix, xxi.

$\overline{\text { VIII }: ~} N, A_{1}, A_{2}^{\prime \prime}, A_{6}^{\prime \prime}, M_{1}, M_{2}^{\prime \prime \prime}, M_{8}, M_{3}^{\prime}, M_{4}, M_{6}$.

$K \overline{\mathrm{VIII}}: \mathrm{i}, \mathrm{x}, \mathrm{xiii}, \mathrm{xx}, \mathrm{v}, \mathrm{vi}$, vii, viii, ix, xxi.

$\overline{\mathrm{IX}}: \mathrm{N}, \mathrm{A}_{1}, \mathrm{~A}_{7}, \mathrm{M}_{1}, \mathrm{M}_{6}, \mathrm{M}_{6}$.

$K \overline{\mathrm{IX}}: \mathrm{i}, \mathrm{x}, \mathrm{xx}, \mathrm{v}, \mathrm{xvi}, \mathrm{xxi}$.

In $K \overline{\mathrm{S}}_{1}, \mathrm{~A}_{2}$ fails for $a=1, b=c=2 ; \mathrm{A}_{3}$ for $a=1, b=0 ; \mathrm{M}_{2}$ for $a=\alpha$, $b=\beta, c=\gamma ; \mathrm{M}_{3}$ for $a=b=1, c=\alpha ; \mathrm{M}_{3}^{\prime}$ for $a=1, b=c=\alpha$.

In $K \overline{\mathrm{S}}^{\prime}, \mathrm{A}_{2}$ fails for $a=1, b=c=2 ; \mathrm{M}_{2}$ for $a=\alpha, b=\beta, c=\gamma ; \mathrm{M}_{3}$ for $a=b=c$ $=1 ; \mathrm{M}_{8}^{\prime}$ for $a=b=c=1$.

In $K \overline{\mathrm{S}}^{\prime \prime}, \mathrm{A}_{6}$ fails for $a=1, b=0 ; \mathrm{M}_{3}$ for $a=b=c=1$.

In $K \mathrm{I}, \mathrm{A}_{2}$ fails for $a=1, b=c=2 ; \mathrm{A}_{3}^{\prime}$ for $a=1, b=0 ; \mathrm{M}_{2}$ for $a=\alpha, b=\beta$, $c=\gamma ; \mathrm{M}_{8}$ for $a=b=1, c=\alpha ; \mathrm{M}_{3}^{\prime}$ for $a=1, b=c=\alpha$.

In $K \overline{\mathrm{II}}, \mathrm{A}_{2}$ fails for $a=1, b=c=2 ; \mathrm{M}_{2}^{\prime \prime}$ for $a=\alpha, b=\beta ; \mathrm{M}_{3}$ for $a=b=c=1$; $\mathrm{M}_{3}^{\prime}$ for $a=b=c=1$.

In $K \overline{\mathrm{III}}, \mathrm{A}_{2}^{\prime}$ fails for $a=1, b=c=0 ; \mathrm{A}_{6}^{\prime \prime}$ for $a=1, b=0 ; \mathrm{M}_{2}^{\prime}$ for $c \neq a$; $\mathrm{M}_{3}$ for $a=b=c=1$.

In $K \overline{\mathrm{IV}}, \mathrm{A}_{2}$ fails for $a=1, b=c=2 ; \mathrm{A}_{6}^{\prime \prime}$ for $b \neq a ; \mathrm{A}_{6}^{\prime \prime \prime}$ for $b \neq a ; \mathrm{M}_{2}$ for $a=\alpha, b=\beta, c=\gamma ; \mathrm{M}_{3}$ for $a=b=1, c=\alpha ; \mathrm{M}_{3}^{\prime}$ for $a=1, b=c=\alpha$.

In $K \overline{\mathrm{V}}, \mathrm{A}_{2}$ fails for $a=1, b=c=2 ; \mathrm{A}_{6}^{\prime}$ for $b \neq a ; \mathrm{M}_{2}$ for $a=\alpha, b=\beta, c=\gamma$; $\mathrm{M}_{3}$ for $a=b=1, c=\alpha ; \mathrm{M}_{3}^{\prime}$ for $a=1, b=c=\alpha$.

In $K \overline{\mathrm{VI}}, \mathrm{A}_{2}$ fails for $a=1, b=c=2 ; \mathrm{A}_{7}$ for $a=0, b=c=1 ; \mathrm{M}_{2}$ for $a=\alpha$, $b=\beta, c=\gamma ; \mathrm{M}_{3}$ for $a=b=1, c=\alpha ; \mathrm{M}_{3}^{\prime}$ for $a=1, b=c=\alpha$.

In $K \overline{\mathrm{VII}}, \mathrm{A}_{2}$ fails for $a=1, b=c=2 ; \mathrm{A}_{3}$ for $b \neq a ; \mathrm{A}_{3}^{\prime \prime}$ for $b \neq a ; \mathrm{M}_{2}$ for $a=\alpha, b=\beta, c=\gamma ; \mathrm{M}_{3}$ for $a=b=1, c=\alpha ; \mathrm{M}_{3}^{\prime}$ for $a=1, b=c=\alpha$.

In $K \overline{V I I I}, \mathrm{~A}_{2}^{\prime \prime}$ fails for $c \neq a ; \mathrm{M}_{2}^{\prime \prime \prime}$ for $a=\alpha, b=\beta, c=\gamma ; \mathrm{M}_{3}$ for $a=b=1$, $c=\alpha ; \mathrm{M}_{3}^{\prime}$ for $a=1, b=c=\alpha$.

In $K \overline{\mathrm{IX}}, \mathrm{A}_{7}$ fails for $a \neq 0, c=0 ; \mathrm{M}_{5}$ for $c \neq e$.

For the independence of Postulate $M_{4}$ in sets $\vec{S}_{1}, \bar{S}^{\prime}, \bar{I}, \overline{I I}, \overline{V I I}$, we may replace system ix by the simpler system xix.

University of California,

Berkeley, Calif. 